\title{
Trait "pessimism" is associated with increased sensitivity to negative feedback in rats
}

\author{
Rafal Rygula $^{1} \cdot$ Piotr Popik ${ }^{1}$
}

Published online: 22 February 2016

(C) Psychonomic Society, Inc. 2016

\begin{abstract}
Several cognitive theories of depression have proposed that cognitive judgment bias determines individual vulnerability to this disorder. Indeed, we have recently demonstrated a relationship between pessimistic judgment bias and vulnerability of rats to the stress-induced anhedonia, and a negative correlation between the level of pessimism and motivation. To further characterize the effects of trait pessimism on cognitive processes associated with depression, in the present study we compared the sensitivity of rats displaying optimistic and pessimistic traits to positive and negative feedback. The animals were initially trained and tested in the rat version of the probabilistic reversal-learning (PRL) task, which allowed for the assessment of feedback sensitivity in individual animals. Subsequently, the rats were re-trained and tested in a series of ambiguous-cue interpretation (ACI) tests, which allowed for the classification of animals displaying "optimistic" and "pessimistic" traits. The "pessimistic" rats were significantly more sensitive to negative feedback than their "optimistic" conspecifics, as indicated by an increased proportion of lose-shift behaviors. The results of our study demonstrate the interrelation and co-existence of two cognitive biases that may predict vulnerability to depressive disorder.
\end{abstract}

Keywords Rat $\cdot$ Ambiguous-cue interpretation $\cdot$ Probabilistic reversal learning $\cdot$ Pessimism $\cdot$ Optimism $\cdot$ Cognitive judgment bias $\cdot$ Feedback sensitivity

Rafal Rygula

rygula@gmail.com

1 Affective Cognitive Neuroscience Laboratory, Department of Behavioral Neuroscience and Drug Development, Institute of Pharmacology, Polish Academy of Sciences, 12 Smetna Street, 31-343 Krakow, Poland

\section{Introduction}

Aberrant cognition has been emphasized in psychological models of mood disorders. In a cognitive model proposed by Aaron Beck (1987), dysfunctional cognitive negative schemata that are activated by stressful life events lead to a characteristic triad of negative thoughts directed at the self, the world, and the future. These thoughts in turn lead to biased judgments and errors of logic that perpetuate a low mood and lead to depression. Clinical evidence has revealed that patients with depression frequently ruminate over their perceived failures and criticism and display an exaggerated response to negative feedback during laboratory testing. A seminal study by Elliott et al. (1997) demonstrated that in cognitive tests, depressed individuals who responded incorrectly on a given trial (trial $\mathrm{N}$ ) were disproportionately likely to fail the subsequent trial $(\mathrm{N}+1)$. Since then, this "catastrophic" response to perceived failure has been shown to impact cognitive ability on any tasks that deliver performancecontingent feedback (Clark et al., 2009).

Recent development of the probabilistic reversal-learning (PRL) task provided an effective way of measuring sensitivity to negative and positive feedback by assessing lose-shift and win-stay behaviors. With this paradigm, it is possible to estimate the probability of switching a response after receiving negative feedback (lose-shifting) and the probability of maintaining a response following positive feedback (win-staying). The probabilistic nature of this task enhances the occurrence of error processing, thus making it particularly suitable for detecting changes in positive and negative feedback sensitivity (Paulus et al., 2002, 2003).

The PRL task is widely used in clinical settings and has been proven effective in detecting changes in sensitivity to feedback in several psychopathologies including depression (Murphy et al., 2003; Taylor Tavares et al., 2008), bipolar 
disorder (Roiser et al., 2009) schizophrenia (Waltz and Gold, 2007), and Parkinson's disease (Cools et al., 2001).

In the present study, we used a preclinical, operant version of this task to evaluate differences in feedback sensitivity between animals displaying "optimistic" and "pessimistic" traits. Our previous studies suggested that these traits might have an influence on animals' motivation (Rygula et al., 2015b) and individual vulnerability to stress-induced anhedonia (Rygula et al., 2013), one of the core symptoms of depression in humans.

To establish individual levels of "optimism"/"pessimism," we subjected the animals to a number of consecutive ambiguous-cue interpretation (ACI) tests (Enkel et al., 2010; Harding et al., 2004; Rygula et al., 2012) performed at 1-week intervals. In the ACI paradigm, rats are trained to press a lever in an operant conditioning chamber to receive a food reward that is contingent on one tone and to press another lever in response to a different tone to avoid punishment by a mild electric foot-shock. The tones, which serve as discriminative stimuli, acquire positive and negative valence, and the training continues until the rats accomplish a stable, correct discrimination ratio. After attaining stable discrimination performance, the animals are tested. Ambiguous cue testing is composed of a discrimination task, as described above, with the presentation of additional tones with intermediate frequencies (between positive and negative tones). The lever press response pattern to these ambiguous-cues is considered an indicator of the rat's expectation of a positive or negative event; in other words, it represents "optimism" or "pessimism," respectively (for details, see Enkel et al., 2010; Papciak et al., 2013; Rygula et al., 2012, 2013). Based on this cognitive bias screening (CBS), the animals were classified into one of two groups: Those displaying a positive cognitive bias towards "optimistic" judgments, referred to as "optimistic," and those displaying a negative cognitive bias towards "pessimistic" judgments, referred to as "pessimistic."

We hypothesized that the traits of "optimism" and "pessimism" would be associated with different sensitivities to positive and negative feedback.

\section{Methods}

\section{Ethics statement}

These experiments were conducted in accordance with the NIH Guide for the Care and Use of Laboratory Animals and were approved by the Ethics Committee for Animal Experiments at the Institute of Pharmacology Polish Academy of Sciences. The authors attest that all efforts were made to minimize the number of animals used and their suffering.

\section{Subjects and housing}

Thirty male Sprague Dawley rats (Charles River, Germany), weighing between 175 and $200 \mathrm{~g}$ upon arrival, were used in this study. The rats were housed in groups (four rats/cage; in one of the cages two additional animals did not take part in this experiment) in a temperature-controlled room $\left(21 \pm 1{ }^{\circ} \mathrm{C}\right)$ with 40-50\% humidity under a 12/12-h light/dark cycle (lights on at 06:00 h). In all of the experiments, the rats were mildly food-restricted to approximately $85 \%$ of their freefeeding weights. This was achieved by providing $15-20 \mathrm{~g}$ of food per rat per day (standard laboratory chow). The food restriction began 1 week prior to training. Water was freely available, except during the test sessions. The behavioral procedures and testing were performed during the light phase of the light/dark cycle.

\section{Apparatus}

The behavioral tasks were performed in eight computercontrolled Skinner boxes (Med Associates, St Albans, VT, USA); each box was equipped with light, a speaker, a liquid dispenser (set to deliver $0.1 \mathrm{ml}$ of $20 \%$ sucrose solution), a grid floor through which scrambled electric shocks $(0.2 \mathrm{~mA}$ (PRL task) or $0.5 \mathrm{~mA}$ (ACI task)) could be delivered, and two retractable levers. The levers were located on opposite sides of the feeder. All of the behavioral protocols, including the data acquisition and recordings, were programmed in Med State notation code (Med Associates). The experimental procedures for the PRL test were modified from procedures described by Bari and colleagues (2010). The experimental procedures for the ACI test used in this study were modified from the procedures previously described by Enkel and colleagues (2010) and have been described in detail elsewhere (Rygula et al., 2015b, 2012).

\section{Behavioral training for the probabilistic reversal-learning (PRL) task}

The animals were trained to perform the probabilistic reversallearning task in several stages. The training and testing sessions lasted for $30 \mathrm{~min}$ or 120 completed trials. Initially, the rats were trained to press the levers located on both sides of the feeder to receive a reward $(0.1 \mathrm{ml}$ of $20 \%$ sucrose solution). Reliable active lever pressing for the reward was achieved in 3 training steps: (a) presentation of the reward for $5 \mathrm{~s}$ every $10 \mathrm{~s}$ for $30 \mathrm{~min}$, which trained the animals to recognize and collect the reward; (b) training to associate lever pressing with receiving a reward - at this stage, pressing any lever resulted in the presentation of the reward; and (c) introduction of the intertrial interval after pressing a lever, during which the levers were retracted and the house light was switched off for $3 \mathrm{~s}$ before a new trial began. If an animal did not press any lever 
within $10 \mathrm{~s}$, the trial was ended and marked as an omission. The training sessions continued until the animals attained a stable performance on each of the training steps (more than 200 responses maintained over three consecutive training sessions during step (b), and a minimum of $90 \%$ of responses to both levers maintained over three consecutive sessions during step (c)). To avoid side bias, we applied additional criteria that animals had to press both levers with similar frequency and a maximal deviation should not exceed $10 \%$ for three consecutive training days.

\section{PRL test}

In the last phase of training, the probabilistic reversal-learning task was introduced (see Fig. 1a for a schematic overview of the task). At the start of the trial, both levers were presented. Pressing one of the levers (the "correct lever") was associated with a high probability of receiving a reward $(0.1 \mathrm{ml} 20 \%$ sucrose solution) and a low probability of receiving a punishment (mild foot shock $0.2 \mathrm{~mA}$ lasting $1 \mathrm{~s}$ ). In contrast, pressing the other lever (the "incorrect lever") was associated with a high probability of receiving the punishment and only a small chance of receiving the reward. The probabilities of receiving the reward and punishment upon pressing the "correct lever" were set to $80 \%$ and $20 \%$, respectively, and vice versa for the opposite "incorrect lever."

The animals had to adjust their behavior by responding to the appropriate levers to maximize reward and minimize punishment delivery, disregarding the occasional misleading positive or negative feedback. After every eight consecutive

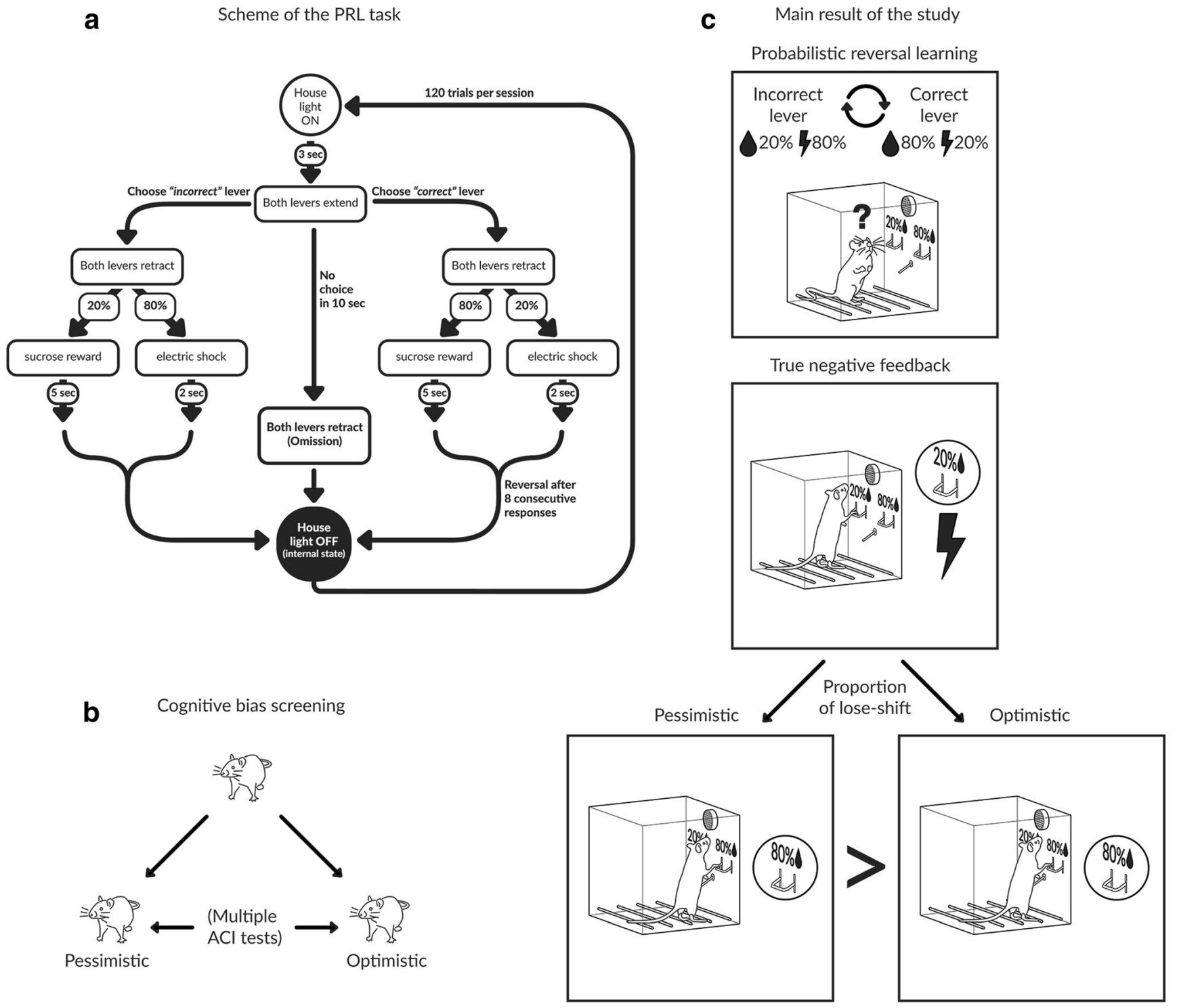

Fig. 1 Experimental design and graphic abstract of the study. 
presses of the "correct lever," the reversal criterion was reached, and the probabilities were reversed; that is, the "correct lever" became the "incorrect lever," and vice versa.

This last training phase was repeated daily until the rats reached the criterion of a minimum of three reversals completed during three consecutive PRL sessions. The animals that successfully accomplished the training were subsequently tested in the PRL procedure ten times across 10 consecutive days.

\section{Behavioral parameters measured in the PRL test}

The main measures of the rats' performance were the total number reversals made during the experimental session and the proportion of responses on the "correct lever." Additionally, Win-Stay/Lose-Shift (WSLS) behavior was analyzed according to the outcome of each preceding trial to assess the sensitivity of the animals to true and misleading positive and negative feedback. The win-stay ratio represents the number of times the subject decided to press the same lever again after it resulted in a reward in the previous trial divided by the total number of rewarded trials. This measure can be interpreted as the sensitivity to positive feedback. The lose-shift ratio is calculated by the number of trials in which the animal decides to change to the other lever in response to a punishment in the previous trial divided by the total number of punishment trials. The lose-shift ratio represents the sensitivity to negative feedback. We analyzed the win-stay and loseshift behaviors separately for true and misleading probabilistic trials. The number of reversals completed was used in the analysis to assess the general performance of the animals on the task, which can be considered as a measure of cognitive flexibility.

\section{Behavioral training for the ambiguous-cue interpretation (ACI) test}

After training and testing animals in the PRL test, the rats were re-trained and re-tested in the ACI paradigm. As mentioned previously, the experimental training and testing procedures for the ACI paradigm used in this study have been described previously (Enkel et al., 2010; Rygula et al., 2015b, 2012).

Initially, the animals were trained to press one lever when a "positive" tone $(2000 \mathrm{~Hz}$ at $75 \mathrm{~dB})$ signaled the reward $(20 \%$ sucrose solution) availability and to press a second lever when another "negative" tone $(9000 \mathrm{~Hz}$ at $75 \mathrm{~dB})$ signaled a forthcoming punishment $(0.5 \mathrm{~mA}, 10 \mathrm{~s})$. By pressing the appropriate levers, the animals could either receive a reward or avoid a punishment. The tone presentations were separated by 10 -s intertrial intervals (ITIs), and each training session lasted $30 \mathrm{~min}$. The animals had to fulfill the criteria of at least $90 \%$ accurate responses to the tone signaling reward availability maintained over three consecutive training sessions and at least $60 \%$ of correct punishment-prevention responses maintained over three consecutive training sessions to proceed to the discrimination training.

During the discrimination-training phase, the animals were trained to discriminate between pseudo-randomly presented positive (20) and negative (20) tones, by pressing the appropriate levers (as learned in the previous training stages) to minimize punishment and maximize reward delivery. Each discrimination training session lasted $40 \mathrm{~min}$. The animals had to achieve a minimum of $70 \%$ correct responses for each lever, maintained over three consecutive discrimination sessions to qualify for the ACI testing.

\section{ACI testing}

During the $\mathrm{ACI}$ testing sessions, the animals were exposed to 20 negative, 20 positive, and ten ambiguous $(5,000 \mathrm{~Hz}$ at $75 \mathrm{~dB}$ ) tone presentations. Each test session lasted $50 \mathrm{~min}$. The tones were played in a pseudo-randomized order and were separated by 10 -s ITIs. The responses to each tone (positive, ambiguous, and negative) during the ACI testing were analyzed as the proportion of the overall number of responses to a given tone. To calculate the cognitive bias index, we subtracted the proportion of negative responses to the ambiguous cues from the proportion of positive responses to the ambiguous cues, which resulted in values ranging between -1 and 1 . Values above 0 indicated an overall positive judgment and "optimistic" interpretation of the ambiguous cue, while the values below 0 indicated overall negative judgment and "pessimism."

\section{Cognitive bias screening (CBS)}

The CBS procedure has been described in detail elsewhere (Rygula et al., 2013). In brief, to assess the cognitive judgment bias as a trait, we examined the animals in a series of ten consecutive ACI tests conducted at 1-week intervals. Based on the average (AVG) cognitive bias index obtained from these ten ACI tests, the rats were divided into two subgroups: "optimistic" and "pessimistic."

\section{Experimental design}

The experimental design is schematically presented in Fig. 1. After attaining the criterion of a minimum of three reversals completed during three consecutive PRL sessions, the animals that successfully accomplished the training were tested ten times in the PRL procedure over 10 consecutive days and were subsequently re-trained for the ACI procedure. After attaining a stable discrimination performance, each rat was subjected to the cognitive bias screening as previously described. After establishing the "optimistic" and "pessimistic" traits in the individual animals, the rats were divided into two 
experimental groups: "optimistic" and "pessimistic" (Fig. 1b). To assess whether the traits of "optimism" and "pessimism" interact with sensitivity to feedback, we compared "optimistic" and "pessimistic" animals for their average performance on the ten PRL tests and average proportion of win-stay and lose-shift behaviors following normal and spurious positive and negative feedback (Fig. 1c).

It is worth noting that the PRL testing needed to be performed before the ACI testing because only in that order would the levers remain unbiased for the second test. If the rats were tested first in the ACI paradigm, the levers would acquire positive and negative meaning by association with a palatable reward or punishment and could not be used as reliable operands in the PRL paradigm.

\section{Statistics}

The data were analyzed using SPSS (version 21.0, SPSS Inc., Chicago, IL, USA). The distribution of the cognitive bias index data was tested using the Kolmogorov-Smirnov test. The difference between "optimists" and "pessimists" in the frequency of "optimism" was analyzed using a t-test. The differences in the processing of the ambiguous cue and the positive and negative tones between the "optimistic" and "pessimistic" animals were investigated using four-way analysis of variance (ANOVA) with the between-subject factor of Cognitive bias (two levels: optimistic and pessimistic) and the withinsubjects factors of Test (ten levels: baseline test 1-10), Lever (two levels: positive and negative) and Tone (three levels: positive, ambiguous and negative). The differences between "optimistic" and "pessimistic" animals in the number of reversals and sensitivity to positive and negative feedback were analyzed separately using t-tests.

The proportions of the "correct lever" presses, "incorrect lever" presses, and omissions were analyzed separately using Mann-Whitney tests, as the data were not normally distributed. For pair-wise comparisons, the values were adjusted using Sidak's correction factor for multiple comparisons (Howell, 1997). All of the tests of significance were performed at $\alpha=$ 0.05 . Homogeneity of variance was confirmed using Levene's test. For repeated-measures analyses, the sphericity was also verified using Mauchly's test. The data are presented as the mean $\pm \mathrm{SEM}$.

\section{Results}

All trained animals reached the criterion of a minimum of three reversals completed during three consecutive PRL sessions and qualified for the PRL testing. Across the ten PRL tests, all animals completed an average $4.39 \pm 0.15$ reversals/ session. The average proportions of win-stay behaviors following true and misleading feedback were $0.86 \pm 0.01$ and
$0.75 \pm 0.01$, respectively. The average proportions of loseshift behaviors following true and misleading feedback were $0.58 \pm 0.01$ and $0.46 \pm 0.02$, respectively. The average proportions of "correct lever" presses, "incorrect lever" presses and omitted trials were $0.29 \pm 0.01,0.65 \pm 0.01$ and $0.06 \pm$ 0.013 , respectively. To compare sensitivity with feedback between "optimistic" and "pessimistic" animals after the PRL testing, the rats were re-trained for the ACI paradigm and classified as "optimistic" or "pessimistic" based on the results of the cognitive bias screening procedure. Twenty-nine out of the 30 trained rats reached the criterion of at least $70 \%$ correct responses with each lever, maintained over three consecutive discrimination sessions, and qualified for the cognitive bias screening. The animals that were classified as "optimistic" reached the criteria of positive tone, negative tone, and discrimination trainings after $2 \pm 0,25.2 \pm 2.2$, and $54.0 \pm$ 5.5 days, respectively, whereas the "pessimistic" group reached the criteria after $2 \pm 0,26.2 \pm 1.8$, and $55.9 \pm 5.8$ days, respectively. Notably, as the animals were already trained to press a lever to obtain a reward, the positive tone training was limited to only two training sessions. No significant differences were observed in the total duration of the training between the "optimistic" and "pessimistic" animals $\left(\mathrm{t}_{(28)}=0.33\right.$, not significant (NS)).

The average cognitive bias index of all of the experimental animals established based on the cognitive bias screening was $0.07 \pm 0.07$. The distribution of the cognitive bias index data during cognitive bias screening was normal $(\mathrm{Z}=0.441, \mathrm{~N}=$ 29, Kolmogorov-Smirnov test).

An analysis of the response of animals to the positive and negative levers following reference and ambiguous tones across the screening period indicated no test-retest effects. Although the Test $\times$ Lever $\times$ Tone interaction was significant $\left(\mathrm{F}_{(18,486)}=2.34, \mathrm{p}<0.001\right)$, post-hoc pairwise comparisons revealed that between-test differences showed no unequivocal patterns.

\section{"Optimistic" versus "pessimistic" animals}

The results of the cognitive bias screening enabled the separation of the animals into two groups that were clearly distinct in their interpretation of the ambiguous cues over time: "pessimistic" $(\mathrm{N}=12, \mathrm{AVG}$ cognitive bias index $<0)$ and "optimistic" ( $\mathrm{N}=17, \mathrm{AVG}$ cognitive bias index $>0)$. Further analysis revealed significant differences in the patterns of response between "pessimistic" and "optimistic" groups (significant Lever $\times$ Tone $\times$ Cognitive bias interaction $\left.\left(\mathrm{F}_{(2,54)}=34.96, \mathrm{p}<0.001\right)\right)$. The "pessimistic" animals responded significantly more often to the negative lever and less often to the positive lever than their "optimistic" counterparts $(p<0.001$, Fig. $2 b$ and $c)$. No significant differences between the "pessimistic" and "optimistic" animals were observed in response to the reference tones 


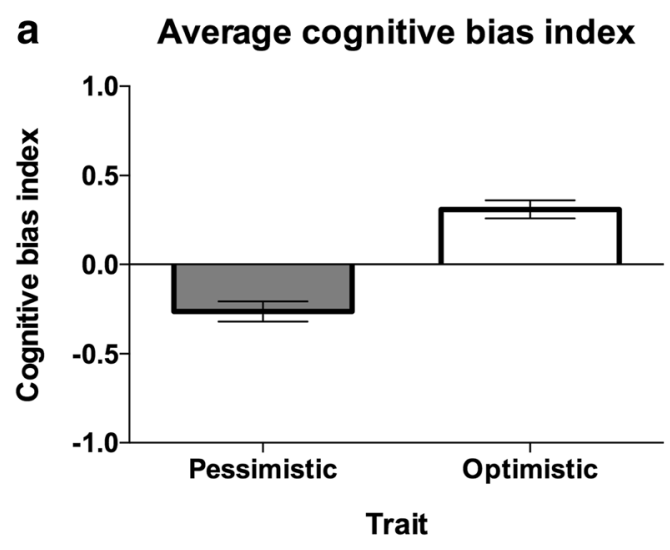

Lever presses

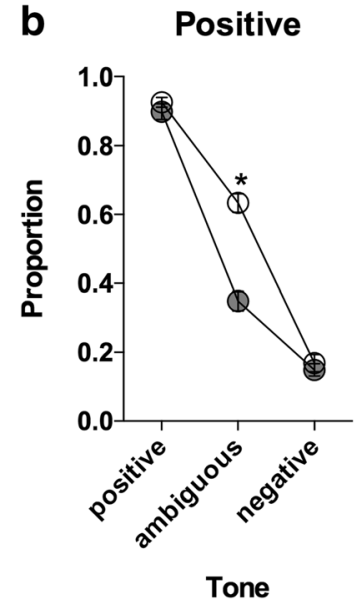

Fig. 2 "Optimistic" versus "pessimistic" animals; results of the cognitive bias screening. a The mean \pm SEM cognitive bias index of the animals classified (based on ten ambiguous cue interpretation (ACI) tests) as "pessimistic" (filled bar, $\mathrm{N}=12$ ) versus "optimistic" (open bar, $\mathrm{N}=$ 17). A cognitive bias index above 0 indicates an overall positive judgment and "optimistic" interpretation of the ambiguous cue. b The

(Fig. 2b and c). "Pessimistic" and "optimistic" rats did not differ in the numbers of omissions they made (no significant effect of Cognitive bias or Cognitive bias $\times$ Tone interaction (Fig. 2d)).

The animals that were classified as "pessimistic" had an average cognitive bias index ranging from -0.01 to -0.65 , whereas the cognitive bias index in the "optimistic" group ranged from 0.05 to 0.89 (Fig. 3a). An analysis of the "optimism" frequency (number of tests when the cognitive bias index of an individual animal was higher than zero, out of the ten cognitive bias screening sessions) revealed that on average, the rats classified as "pessimists" were significantly $(p<0.05)$ less frequently "optimistic" than their "optimistic" conspecifics $\left(\mathrm{t}_{(28)}=7.77\right)$ (Fig. $3 \mathrm{a}$ - inset).

Although, as mentioned previously, the cognitive bias index fluctuated in both groups of animals (significant Test $\times$ Lever $\times$ Tone interaction $\left(\mathrm{F}_{(18,486)}=2.94, \mathrm{p}<\right.$
Negative

d Omissions

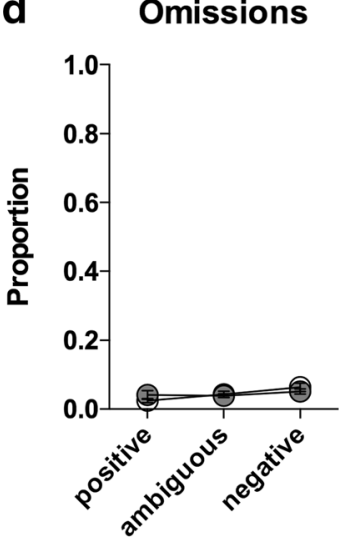

Tone

Tone

mean \pm SEM proportions of positive, $\mathbf{c}$ negative, and $\mathbf{d}$ omitted responses to the trained and ambiguous tones in the "pessimistic" (filled circles, $\mathrm{N}=12$ ) and "optimistic" (open circles, $\mathrm{N}=17$ ) rat groups. * Indicates significant $(\mathrm{p}<0.05)$ differences between the "optimistic" and "pessimistic" animals

0.001)), the differences between the "pessimistic" and "optimistic" groups did not change significantly across the screening period (no significant Test $\times$ Cognitive bias interaction; $F_{(9,243)}=0.63$, NS), indicating a stability of the traits (Fig. 3b).

\section{Effects of cognitive judgment bias on rats' performance in the PRL test}

In the PRL task, no significant differences between the "optimistic" and "pessimistic" animals were observed in the average number of reversals made per experimental session (Fig. $4 a, t_{(28)}=1.29$, NS) nor in the proportions of winstaying following true reward feedback (Fig. $4 b, t_{(28)}=1.41$, NS). However, "pessimism" was associated with a significantly higher sensitivity to negative feedback than "optimism" (Fig. 4c). A t-test revealed that the average proportion of 


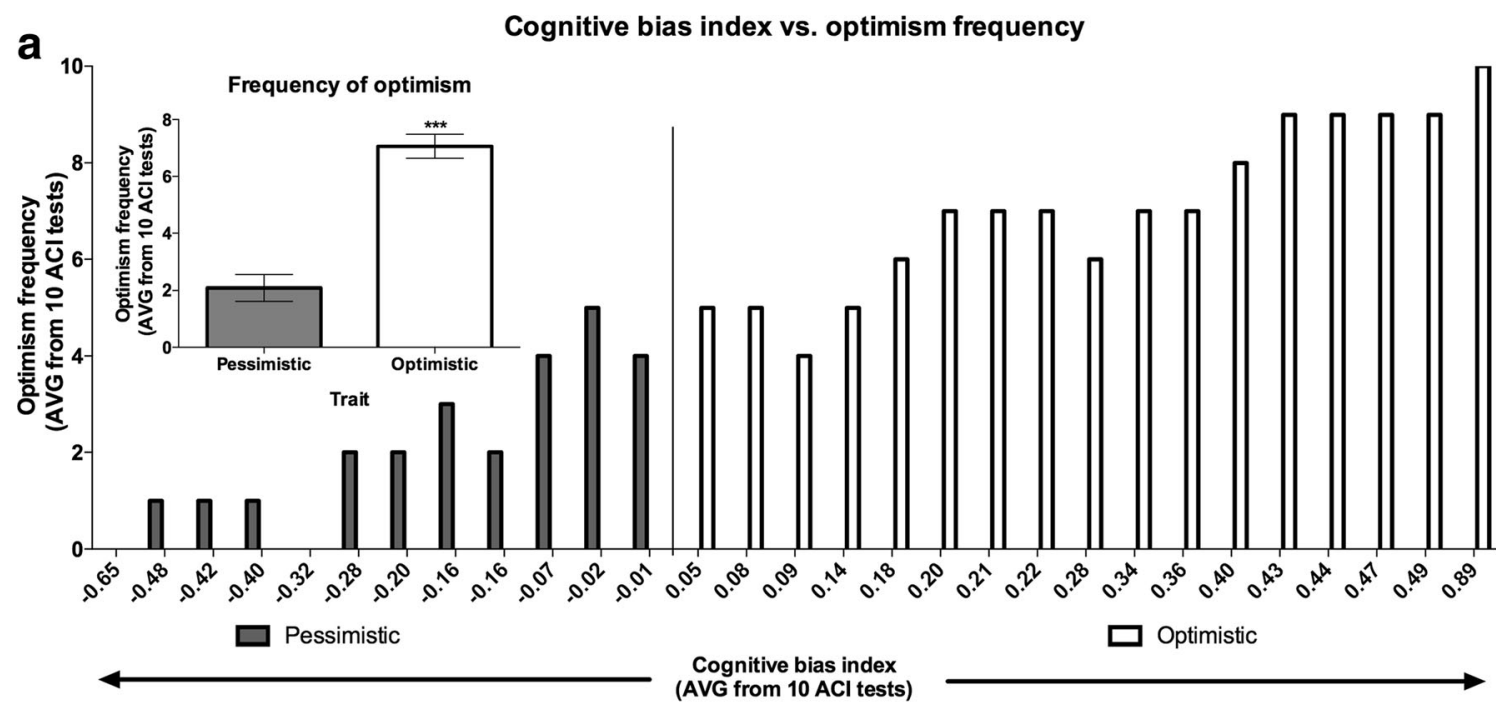

b Cognitive judgement bias across $10 \mathrm{ACl}$ tests
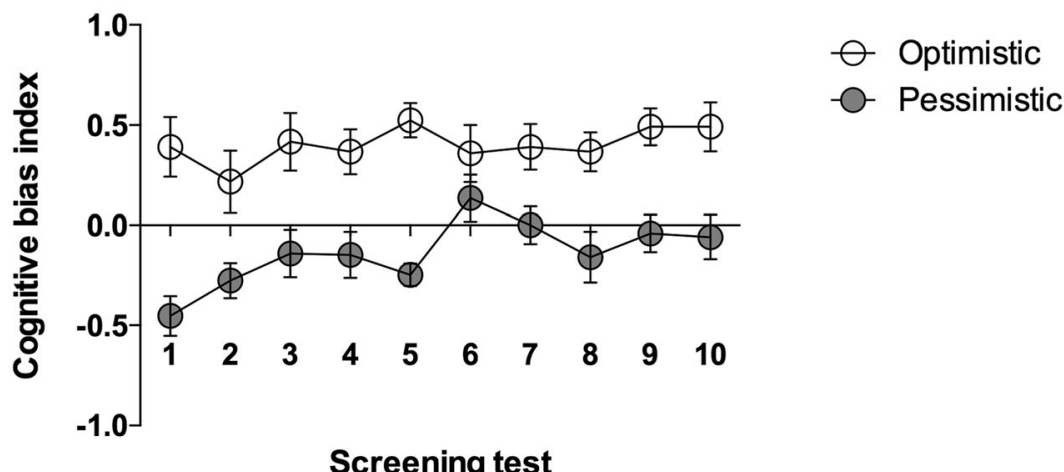

\section{Screening test}

Fig. 3 Cognitive bias as a stable and enduring behavioral trait. a Histogram of the "optimism" frequency (number of ambiguous cue interpretation (ACI) tests resulting in a cognitive bias index above 0 out of the ten ACI tests comprising the cognitive bias screening) in relation to the valence of individual cognitive bias index (AVG from cognitive bias screening $)$ in all $(\mathrm{N}=29)$ animals. Inset: The mean \pm SEM "optimism"

switching responses after receiving a true punishment (loseshift behaviors) in the "pessimistic" animals was significantly higher than the proportion observed in their "optimistic" counterparts $\left(\mathrm{t}_{(27)}=2.06, \mathrm{p}<0.05\right.$, Fig. $\left.4 \mathrm{c}\right)$. We did not observe any significant differences between the "optimistic" and "pessimistic" animals in the proportions of win-staying and loseshifting behaviors following misleading positive and misleading negative feedback, respectively $\left(\mathrm{t}_{(28)}=0.16\right.$ and 0.33 , respectively, NS; Fig. 4d and e)

The "optimistic" and "pessimistic" animals differed significantly in the proportion of "correct lever" presses (Fig. 5a). A Mann-Whitney test indicated that "pessimistic" animals (median $=0.68)$ pressed the "correct lever" significantly more often than their "optimistic" (median $=0.65$ ) conspecifics $(\mathrm{U}=54.50, \mathrm{p}<0.05)$. A Mann-Whitney test indicated that the "pessimistic" and "optimistic" animals (medians $=0.30$ and 0.29 , respectively) did not significantly differ in their proportions of "incorrect lever" presses $(U=95.50, \mathrm{NS}$; Fig. 5b). frequency of the animals classified (based on ten ACI tests) as "optimistic" (open bars, $\mathrm{N}=17$ ) and "pessimistic" (filled bars, $\mathrm{N}=12$ ). b The mean \pm SEM cognitive bias index of the animals classified as "pessimistic" (filled circles, $\mathrm{N}=12$ ) and "optimistic" (open circles, $\mathrm{N}=$ 17) across all ten baseline ACI tests

We also observed no significant differences in the number of omitted trials (medians $=0.02$ and 0.05 , respectively, $\mathrm{U}=$ 72.50, NS; Fig. 5c).

\section{Discussion}

In the present study, we used a PRL task to investigate how "optimistic" and "pessimistic" animals incorporate feedback (both rewarding and punishing) in their decisions in a changing and uncertain environment where feedback is probabilistic and contingencies change. The results of our study indicated that animals displaying the "pessimistic" trait were more sensitive to negative feedback compared with their "optimistic" conspecifics. In combination with our previous reports (Rygula et al., 2015b, 2013), the results of the present study add to the claim that in rats, "pessimistic" judgment bias as a 
True feedback
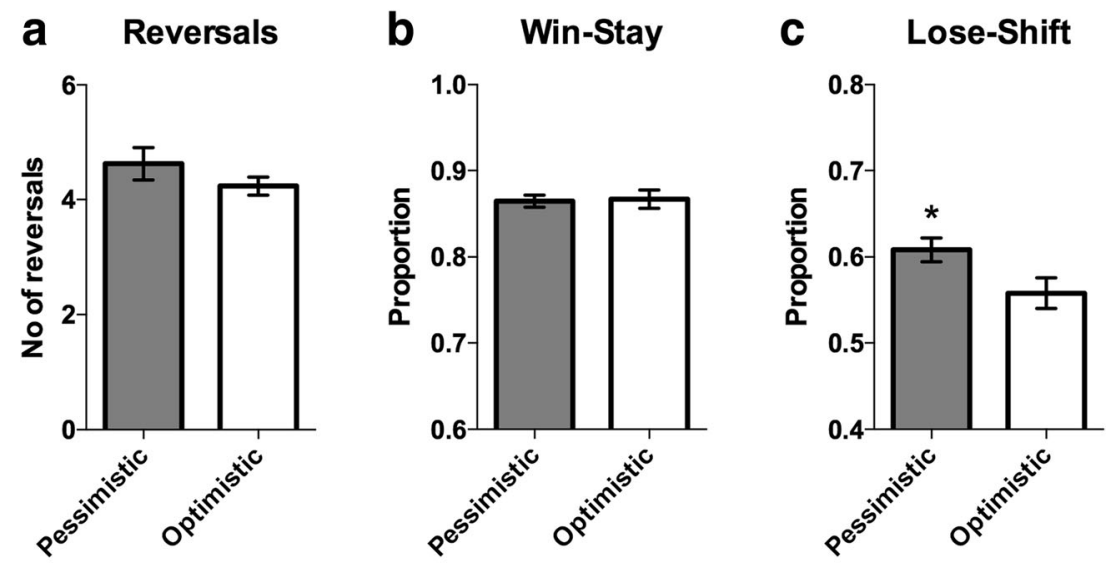

\section{Misleading feedback}
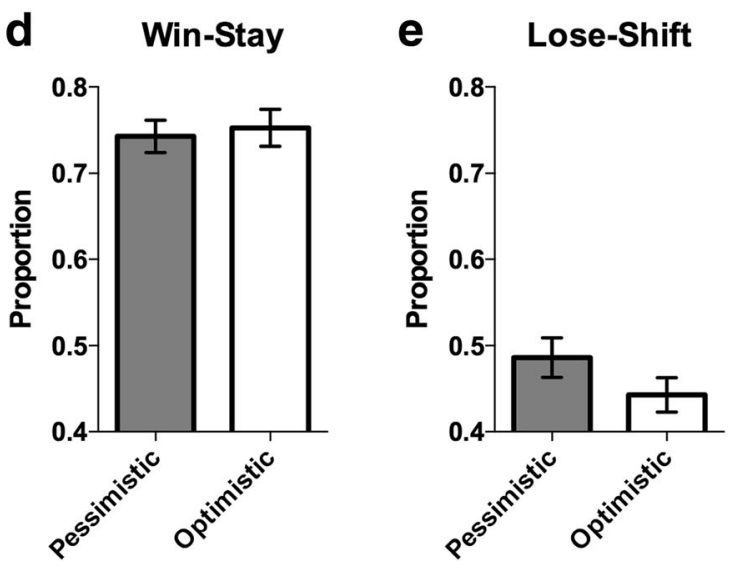

Fig. 4 Probabilistic reversal learning in the "pessimistic" and "optimistic" rats. a The average number of reversals per session, and $\mathbf{b}$ proportions of win-staying and lose-shifting after true (b and c, respectively) and misleading (d and $\mathbf{e}$, respectively) feedback in the animals classified as "pessimistic" (filled bar, $\mathrm{N}=12$ ) and "optimistic" $(\mathrm{N}=17)$. The "pessimists" were significantly $(*, \mathrm{p}<0.05)$ more likely than "optimists" to switch their responding following true negative feedback (panel C)

\section{'Correct lever' presses}

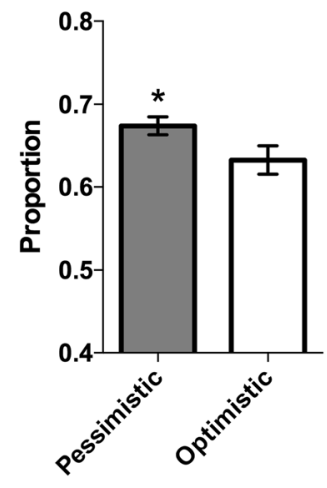

Fig. 5 Proportion of "correct" and "incorrect lever" presses and omitted trials during the PRL tests, in "pessimistic" and "optimistic" animals. The mean \pm SEM proportion of (a) "correct lever" presses, (b) "incorrect lever" presses, and (c) omitted trials in animals classified (based on ten

\section{'Incorrect lever' presses Omissions}
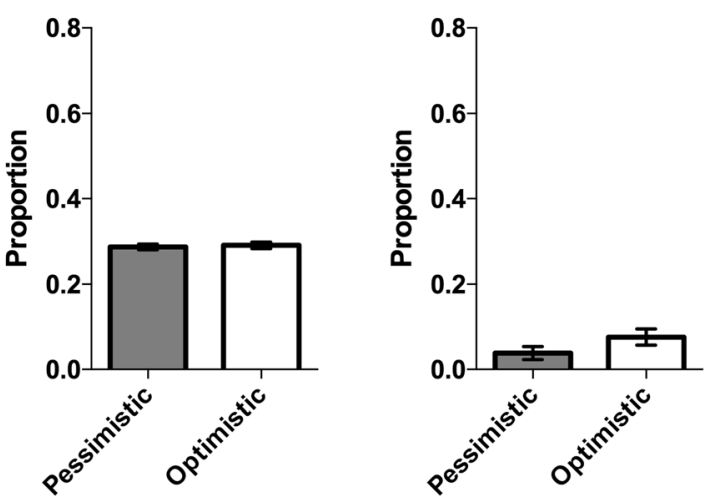

ambiguous cue interpretation (ACI) tests) as "pessimistic" (filled bar, $\mathrm{N}=$ 12) versus "optimistic" (open bar, $\mathrm{N}=17)$. * Indicates significant ( $\mathrm{p}<$ $0.05)$ differences between the "optimistic" and "pessimistic" animals 
trait is linked with a wide spectrum of behavioral and cognitive processes that may be associated with increased vulnerability to depression. Along with increased sensitivity to negative feedback, trait pessimism has also been previously associated with an increased sensitivity to stress-induced anhedonia (Rygula et al., 2013) and decreased motivation (Rygula et al., 2015b).

The valence of cognitive judgment bias as a trait, measured in multiple ACI tests was both quantitative (frequency of "optimism") and qualitative (height of cognitive bias index). An analysis of the lever responses during the tests comprising the cognitive bias screening revealed that the animals classified as "pessimistic" were both more "pessimistic" (made a significantly higher proportion of negative lever presses in response to the ambiguous cue) and less "optimistic" (made a significantly lower proportion of positive lever presses in response to the ambiguous cue) than their "optimistic" conspecifics. This pattern was similar to the one described previously (Rygula et al., 2015b). It is worth noticing that as there were no significant differences between "optimists" and "pessimists" neither in the length of the negative tone training $(\mathrm{t}(28)=0.37, \mathrm{NS})$ nor in the proportion of negative lever presses in response to the negative reference tone (Fig. 2c), the pessimistic judgment bias of animals did not result from the increased sensitivity to electric foot-shocks.

The PRL paradigm has been widely used in studies investigating feedback-related decision-making in humans. These include behavioral (Dickstein et al., 2010; Dombrovski et al., 2010), electrophysiological (Chase et al., 2011), brain imaging (Cools et al., 2002; Hampton \& O'Doherty, 2007) and computational modeling (den Ouden et al., 2013; Glascher et al., 2009; Hampton et al., 2006) approaches. Recently, the PRL paradigm has been introduced into preclinical animal studies. In 2010, Bari and colleagues (2010) demonstrated the effects of different serotonergic (5-HT) manipulations on sensitivity of rats to feedback in the PRL paradigm. Two years later, Ineichen and colleagues (2012) investigated the role of 5-HT on feedback sensitivity in mice, and recently, the PRL paradigm has been employed to investigate the roles of 5-HT and DA in feedback-based decision making in non-human primates (marmosets) (Clarke et al., 2014; Rygula et al., 2015a). It is worth noticing that in the present study we used an operant PRL paradigm with a real punishment (mild foot-shock) for the first time, as opposed to only a lack of reward, as has been used in the previous studies with rats. This increases the translational value of our paradigm, as in humans the PRL tasks consist of rewards and punishments. The use of real punishment in the PRL is also important given the separation of drug treatment effects that they can have when punishment is used versus lack of reward. For example, amphetamine increases impulsivity in rats when the only punishment is lack of reward, while it reduces impulsivity when punishments are used in rats and mice (see Orsini et al., 2015 for review).

A detailed analysis of the responsivity of the "optimistic" and "pessimistic" animals to true and misleading positive and negative feedback revealed that cognitive judgment bias was linked exclusively with true negative feedback sensitivity. "Pessimism" made animals more likely to shift after a true punishment but did not significantly affect their responses after misleading punishment trials during the probabilistic discriminations. This was surprising given that the subjects were not aware of the feedback validity.

Although our experimental protocols did not allow for the separation of acquisition from reversal trials, one explanation for this phenomenon may be that "pessimism" affected the initial phases of contingency learning more when the true negative and positive feedback were dominant, in contrast to later phases, when animals, having established which lever is more "correct," kept pressing it while ignoring the infrequent misleading negative feedback. This explanation would imply that cognitive judgment bias is important in learning from negative feedback but not in ignoring probabilistic errors. Further studies should shed more light on this issue with a detailed analysis of the different phases of probabilistic discrimination in "optimistic" and "pessimistic" animals.

Notably, we also did not observe any significant differences between the "optimists" and "pessimists" in their general performance of the PRL task, as expressed by a similar number of reversals made per experimental session. We hypothesize that the increased sensitivity to negative feedback observed in trials with true punishment could have improved general performance of the task, but this effect was hampered by the effects of a (statistically insignificant) increased proportion of lose-shifting on trials following a misleading punishment (Fig. 4e). This hypothesis is also supported by an analysis of the proportion of "correct" and "incorrect" lever presses. The "pessimistic" animals pressed the "correct lever" significantly more often than the "optimists," although this did not translate to an increased number of reversals. Such a pattern of results suggests that pessimistic animals, although better at switching their responses after a true punishment, also did more (statistically insignificant) undesired lose-shifting following a misleading punishment that disrupted their ability to fulfill the criterion and reverse.

Taken together, using an operant version of the PRL paradigm and multiple consecutive ACI tests, we demonstrate for the first time a link between cognitive judgment 
bias and sensitivity to performance feedback in an animal model. Based on the combination of the present results and our previous reports, one could predict that cognitive judgment bias would be a powerful shaper of the depressive-like behavioral phenotype, not only in laboratory animals but also in other species, including humans. Evidence of this may be useful for developing new therapeutic strategies that are based on early screening for pessimistic judgment biases that may predict further vulnerability to depression. Such a screening would allow preventive therapeutic intervention (e.g., cognitive behavioral therapy, which focuses on accentuating positivity and minimizing negative sensitivity (Beck, 1991)) even before the onset of first depressive symptoms.

Acknowledgments This work was supported by the Polish National Science Center (Research grant DEC-2014/13/B/NZ4/00214 to RR) and the statutory funds of the Institute of Pharmacology Polish Academy of Sciences.

Author contributions We would like to thank Jakub Kubik for his technical assistance in performing the behavioral experiments and Marcin Rzycki for graphical support.

RR conceived and designed the experiments, RR and JK performed the experiments, RR analyzed the data, RR and PP contributed materials/ analysis tools, RR wrote the paper, and RR and PP revised the paper critically for important intellectual content and gave final approval of the version to be published.

\section{Compliance with ethical standards}

Conflicts of interest The authors declare that there are no conflicts of interest in this study.

\section{References}

Bari, A., Theobald, D. E., Caprioli, D., Mar, A. C., Aidoo-Micah, A., Dalley, J. W., Robbins, T. W. (2010). Serotonin modulates sensitivity to reward and negative feedback in a probabilistic reversal learning task in rats. Neuropsychopharmacology, 35(6), 1290-1301.

Beck, A. T. (1987). Cognitive models of depression. Journal of Cognitive Psychotherapy, 1, 5-37.

Beck, A. T. (1991). Cognitive therapy. A 30-year retrospective. The American Psychologist, 46(4), 368-375.

Chase, H. W., Swainson, R., Durham, L., Benham, L., \& Cools, R. (2011). Feedback-related negativity codes prediction error but not behavioral adjustment during probabilistic reversal learning. Journal of Cognitive Neuroscience, 23(4), 936-946.

Clark, L., Chamberlain, S. R., \& Sahakian, B. J. (2009). Neurocognitive mechanisms in depression: implications for treatment. Annual Review of Neuroscience, 32, 57-74.

Clarke, H. F., Cardinal, R. N., Rygula, R., Hong, Y. T., Fryer, T. D., Sawiak, S. J., ... Roberts, A. C. (2014). Orbitofrontal dopamine depletion upregulates caudate dopamine and alters behavior via changes in reinforcement sensitivity. The Journal of Neuroscience, 34(22), 7663-7676.
Cools, R., Barker, R. A., Sahakian, B. J., \& Robbins, T. W. (2001). Enhanced or impaired cognitive function in Parkinson's disease as a function of dopaminergic medication and task demands. Cerebral Cortex, 11(12), 1136-1143.

Cools, R., Clark, L., Owen, A. M., \& Robbins, T. W. (2002). Defining the neural mechanisms of probabilistic reversal learning using eventrelated functional magnetic resonance imaging. The Journal of Neuroscience, 22(11), 4563-4567.

den Ouden, H. E., Daw, N. D., Fernandez, G., Elshout, J. A., Rijpkema, M., Hoogman, M., ... Cools, R. (2013). Dissociable effects of dopamine and serotonin on reversal learning. Neuron, 80(4), 1090-1100.

Dickstein, D. P., Finger, E. C., Brotman, M. A., Rich, B. A., Pine, D. S., Blair, J. R., Leibenluft, E. (2010). Impaired probabilistic reversal learning in youths with mood and anxiety disorders. Psychological Medicine, 40(7), 1089-1100.

Dombrovski, A. Y., Clark, L., Siegle, G. J., Butters, M. A., Ichikawa, N., Sahakian, B. J., Szanto, K. (2010). Reward/Punishment reversal learning in older suicide attempters. The American Journal of Psychiatry, 167(6), 699-707.

Elliott, R., Sahakian, B. J., Herrod, J. J., Robbins, T. W., \& Paykel, E. S. (1997). Abnormal response to negative feedback in unipolar depression: evidence for a diagnosis specific impairment. Journal of Neurology, Neurosurgery, and Psychiatry, 63(1), 74-82.

Enkel, T., Gholizadeh, D., von Bohlen Und Halbach, O., Sanchis-Segura, C., Hurlemann, R., Spanagel, R., ... Vollmayr, B. (2010). Ambiguous-cue interpretation is biased under stress- and depression-like states in rats. Neuropsychopharmacology, 35(4), $1008-1015$.

Glascher, J., Hampton, A. N., \& O'Doherty, J. P. (2009). Determining a role for ventromedial prefrontal cortex in encoding action-based value signals during reward-related decision making. Cerebral Cortex, 19(2), 483-495.

Hampton, A. N., Bossaerts, P., \& O'Doherty, J. P. (2006). The role of the ventromedial prefrontal cortex in abstract state-based inference during decision making in humans. The Journal of Neuroscience, 26(32), 8360-8367.

Hampton, A. N., \& O'Doherty, J. P. (2007). Decoding the neural substrates of reward-related decision making with functional MRI. Proceedings of the National Academy of Sciences of the United States of America, 104(4), 1377-1382.

Harding, E. J., Paul, E. S., \& Mendl, M. (2004). Animal behaviour: cognitive bias and affective state. Nature, 427(6972), 312.

Howell, D. C. (1997). Statistical methods for psychology (4th ed.). Belmont: Wadsworth.

Ineichen, C., Sigrist, H., Spinelli, S., Lesch, K. P., Sautter, E., Seifritz, E., Pryce, C. R. (2012). Establishing a probabilistic reversal learning test in mice: evidence for the processes mediating reward-stay and punishment-shift behaviour and for their modulation by serotonin. Neuropharmacology, 63(6), 1012-1021.

Murphy, F. C., Michael, A., Robbins, T. W., \& Sahakian, B. J. (2003). Neuropsychological impairment in patients with major depressive disorder: the effects of feedback on task performance. Psychological Medicine, 33(3), 455-467.

Orsini, C. A., Moorman, D. E., Young, J. W., Setlow, B., \& Floresco, S. B. (2015). Neural mechanisms regulating different forms of riskrelated decision-making: Insights from animal models. Neuroscience and Biobehavioral Reviews, 58, 147-167.

Papciak, J., Popik, P., Fuchs, E., \& Rygula, R. (2013). Chronic psychosocial stress makes rats more 'pessimistic' in the ambiguous-cue interpretation paradigm. Behavioural Brain Research, 256, 305-310.

Paulus, M. P., Hozack, N., Frank, L., \& Brown, G. G. (2002). Error rate and outcome predictability affect neural activation in prefrontal cortex and anterior cingulate during decision-making. NeuroImage, 15(4), 836-846.

Paulus, M. P., Hozack, N., Frank, L., Brown, G. G., \& Schuckit, M. A. (2003). Decision making by methamphetamine-dependent subjects 
is associated with error-rate-independent decrease in prefrontal and parietal activation. Biological Psychiatry, 53(1), 65-74.

Roiser, J. P., Cannon, D. M., Gandhi, S. K., Taylor Tavares, J., Erickson, K., Wood, S., ... Drevets, W. C. (2009). Hot and cold cognition in unmedicated depressed subjects with bipolar disorder. Bipolar Disorders, 11(2), 178-189.

Rygula, R., Clarke, H. F., Cardinal, R. N., Cockcroft, G. J., Xia, J., Dalley, J. W., ... Roberts, A. C. (2015a). Role of Central Serotonin in Anticipation of Rewarding and Punishing Outcomes: Effects of Selective Amygdala or Orbitofrontal 5-HT Depletion. Cerebral Cortex, 25(9), 3064-3076.

Rygula, R., Golebiowska, J., Kregiel, J., Kubik, J., \& Popik, P. (2015b). Effects of optimism on motivation in rats. Frontiers in Behavioral Neuroscience, 9, 32.
Rygula, R., Papciak, J., \& Popik, P. (2013). Trait pessimism predicts vulnerability to stress-induced anhedonia in rats. Neuropsychopharmacology, 38(11), 2188-2196.

Rygula, R., Pluta, H., \& Popik, P. (2012). Laughing rats are optimistic. PloS One, 7(12), e51959.

Taylor Tavares, J. V., Clark, L., Furey, M. L., Williams, G. B., Sahakian, B. J., \& Drevets, W. C. (2008). Neural basis of abnormal response to negative feedback in unmedicated mood disorders. NeuroImage, 42(3), 1118-1126.

Waltz, J. A., \& Gold, J. M. (2007). Probabilistic reversal learning impairments in schizophrenia: further evidence of orbitofrontal dysfunction. Schizophrenia Research, 93(1-3), 296-303. 